Ann. Zootech., I980, 29 (I), 87-9I.

\title{
Note
}

\section{Attente à l'abattoir et viandes à coupe sombre chez les bovins}

\author{
G. MONIN et P. ROYANT* \\ Station de Recherches sur la Viande, \\ Centre de Recherches de Clermont-Ferrand, I.N.R.A., \\ Theix, St-Genès-Champanelle, 63iтo Beaumont (France)
}

\section{Résumé}

Des observations réalisées dans un abattoir commercial sur 380 taurillons montrent que dans le cas de transports courts (inférieurs à une heure et demie), le délai écoulé entre le départ de la ferme pour l'abattoir et l'abattage influence très fortement la proportion de viandes à $\mathrm{pH}$ élevé $(\mathrm{pH}>6,0)$. Il est donc nécessaire, lorsque l'abattoir ne dispose que d'installations de parquage collectif, d'abattre les animaux dès que possible après leur arrivée à l'abattoir.

\section{Introduction}

Les viandes à coupe sombre sont caractérisées d'abord par leur couleur anormalement foncée, mais aussi par leur pouvoir de rétention d'eau très élevé qui leur confère une texture " collante " caractéristique. Ces deux particularités résultent d'une augmentation du $\mathrm{pH}$ (qui atteint des valeurs de 6,0 ou davantage) dont l'origine réside dans la déplétion des réserves glycogéniques de la musculature avant l'abattage.

Les causes d'apparition de ce type de viande sont encore assez mal connues. On sait toutefois qu'elles résident dans la fatigue musculaire et les excitations inévitablement occasionnées par les transports et les manipulations précédant l'abattage (LAWRIE, I958). Par ailleurs ces viandes s'observent essentiellement chez les taurillons (FREDEEN, MARTIN et WEISS, I974) et dans la tranche d'âge I6-I8 mois (VALIN et SoRnay, I975). Leur fréquence augmente avec la longueur du transport (VALIN et SORNAY, I975).

(*) Adresse actuelle : Kergroas, 29121 Clohars-Carnoët. 
Dans ce travail, nous avons étudié l'influence du délai s'écoulant entre le moment où l'animal quitte la ferme pour l'abattoir et l'abattage, sur le $\mathrm{pH}$ de la viande de taurillons de diverses races, abattus dans un abattoir commercial, dans les conditions normales de la pratique.

\section{Conditions expérimentales et résultats}

Les observations ont été réalisées pendant le mois de mai I 976 sur 380 animaux de race Française Frisonne Pie Noire (FFPN), Normande, Charolais $\times$ Normande ou Limousin $\times F F P N$, les animaux de pure race laitière représentant 95 p. roo de l'effectif total. Tous les animaux étaient élevés en stabulation libre.

Les animaux étaient transportés de leur élevage d'origine à l'abattoir par camion, la distance maximale étant de cinquante kilomètres, ce qui entraînait un délai d'au plus une heure et demie entre la sortie des parcs d'élevage et l'arrivée à l'abattoir. Chaque camion pouvait contenir un maximum de quatorze animaux. Si ceux-ci provenaient d'un même élevage, ils étaient mélangés pendant le transport, mais une cloison transversale permettait d'éviter le mélange d'animaux provenant de deux élevages différents. A leur arrivée à l'abattoir, les taurillons étaient placés dans des enclos susceptibles de contenir une vingtaine d'animaux; à ce moment, il arrivait que des animaux d'élevages différents soient placés ensemble. Ces enclos n'étaient pas abrités. Les taurillons étaient abattus dans les huit heures suivant leur déchargement. Des mesures de $\mathrm{pH}$ étaient effectuées entre vingt et quarante heures post mortem au centre de la section transversale du muscle Longissimus dorsi, au niveau de la $5^{\mathbf{e}}$ côte. Selon Tarrant (I975), le délai de vingt heures est suffisant pour que le $\mathrm{pH}$ s'abaisse à cette localisation au-dessous de 6,0, valeur retenue comme valeur critique au cours de cette étude. Des essais préliminaires ont d'ailleurs confirmé que les muscles présentant à vingt heures post mortem un pH supérieur à 6 , o ne subissaient pratiquement pas d'abaissement ultérieur du $\mathrm{pH}$.

Les températures extérieures pendant la période des mesures ont été obtenues dans une station météorologique située à dix kilomètres environ de l'abattoir.

La figure IA montre que sur l'ensemble des animaux la fréquence des $\mathrm{pH}$ supérieurs à 6,0 augmente très rapidement avec le délai s'écoulant entre le départ de la ferme et l'abattage. Le phénomène est encore plus net si l'on ne considère que les taurillons de $\mathrm{I} 5$ à $\mathrm{I} 7$ mois pour lesquels on obtient $43 \mathrm{p}$. Ioo de pH élevés lorsque le temps de transport et attente cumulé dépasse 5 heures (fig. IB).

Parmi les animaux observés, un lot comprenait I 6 animaux du même élevage abattus le même jour, représentant donc un matériel très homogène. Les observations réalisées sur ce lot confirment que l'augmentation de la durée d'attente au delà de 5 heures conduit à une nette élévation du $\mathrm{pH}$ de la viande (fig. $\mathrm{IC}$ ).

SORNAY (I978) suggère que les températures élevées favorisent l'apparition de viande à $\mathrm{pH}$ élevé. Nos résultats montrent que l'on peut rencontrer des fréquences importantes de $\mathrm{pH}$ élevés même avec des températures extérieures très modérées et présentant de faibles amplitudes de variation au cours de la journée (fig. 2 ; moyenne des températures à $\mathrm{I} 4$ heures, en excluant le $6 \mathrm{mai}=\mathrm{I}_{3}, 8^{\circ} \mathrm{C}$ ). Cependant l'observation d'une proportion particulièrement élevée le 6 mai (33 p. Ioo de $\mathrm{pH}$ supérieurs à 6,0 contre une moyenne de I9 $\mathrm{p}$. roo pour les autres jours d'abattage), jour où la température était nettement supérieure, tend à 

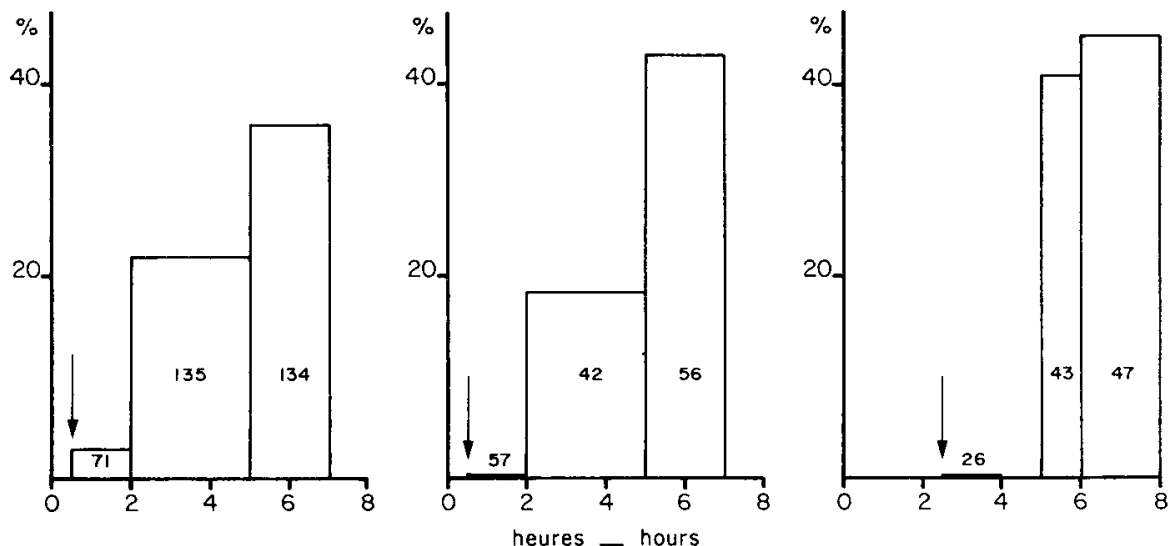

Fíg. I. - Pourcentage d'animaux présentant un $p H>6.0$ dans le muscle Longissimus dorsi en fonction du délai séparant le départ de la ferme de l'abattage.

I $a$ : 340 animaux de 15 à 24 mois, Ib : I 55 animaux de 15 à $I 7$ mois.

Ic : II6 animaux d'un même élevage abattus le même jour (6 Mai).

La fèche $\downarrow$ indique le début des opérations d'abattage.

Les nombres portés sur les figures indiquent le nombre d'animaux abattus dans l'intervalle de temps considéré.

Percentage of animals exhibiting a $p H>6.0$ in the Longissimus dorsi muscle according to the interval (hours) between the departure from the farm and slaughter.

$I a: 340$ animals aged $15-24$ months $-I$ b : I55 animals aged ${ }_{55-17}$ months.

IC : II6 animals from the same herd slaughtered the same day (6 May).

The arrow $\downarrow$ indicates the beginning of slaughter. The figures indicate the number of animals slaughtered within the time interval considered.

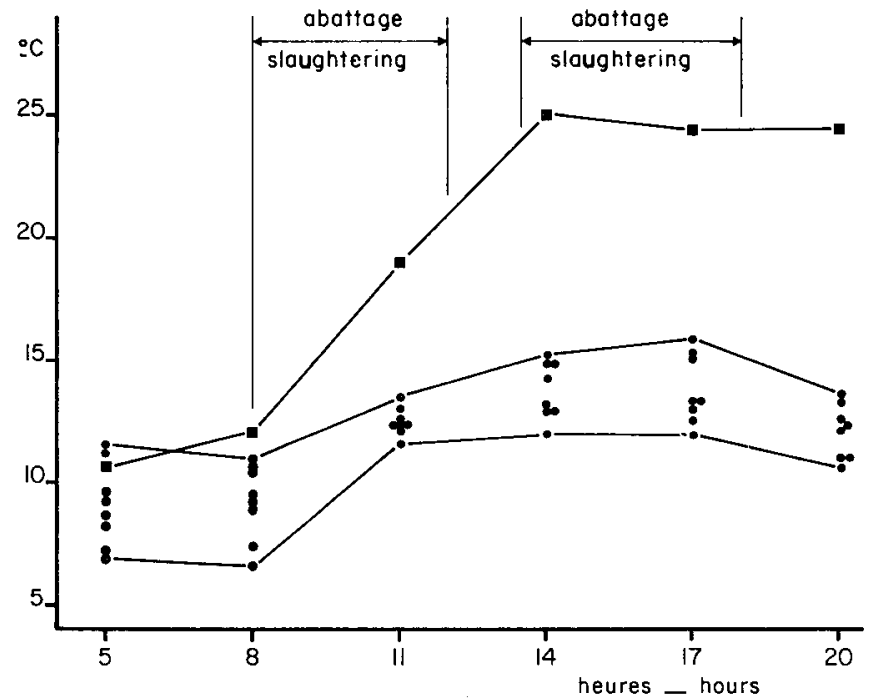

FIG. 2. - Evolution de la température extérieuve les jours d'abattage.

Temperature variations on the days of slaughter.

- 6 mai : abattage du lot de Ir6 bovins d'une même exploitation.

- $6^{\text {th }}$ may : slaughter of II6 animals from the same hevd.

- Autres jours d'abattage.

- Other slaughter days. 
confirmer la conclusion de SORNAY (I978). Sur trois carcasses montrant d'importants hématomes sur la partie dorsale, attribués au chevauchement, on a relevé des valeurs de $\mathrm{pH}$ de $6,4-6,6$ et 7 ,I. I1 semblerait donc que le chevauchement provoque l'obtention de $\mathrm{pH}$ de la viande très élevés chez les animaux qui le subissent, ce qui est en accord avec les observations de RHoDes (I973).

TABI,EAU I

Fréquence des viandes à coupe sombre dans les taurillons abattus Frequency of dark-cutting beef in young bull carcasses

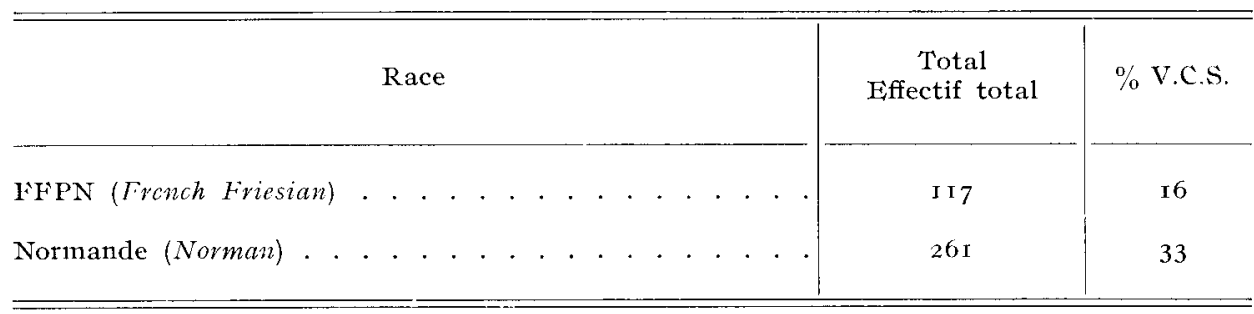

Il existe des différences raciales comme le montre le tableau $r$, les animaux de race Normande présentant une proportion de $\mathrm{pH}$ élevés supérieure à celles des $F F P N$. Ceci ne permet toutefois pas de conclure sur une éventuelle telation entre type laitier et sensibilité au stress, relation d'ailleurs très sujette à controverses (MONIN et VAIIN, I976).

\section{Conclusion}

Les valeurs que nous rapportons pour la fréquence des $\mathrm{pH}$ de la viande supérieurs à 6,o dans l'abattoir observé paraissent très élevées (valeur moyenne d'environ 23 p. IOO) mais ne sont pas exceptionnelles. SorNay (I978) rapporte une proportion de 18 p. Ioo pour I I50 taurillons étudiés par 1'I.T.E.B. et indique coinme fréquente l'obtention de ro à I5 $\mathrm{p}$. Ioo de carcasses à $\mathrm{pH}$ élevé.

Nos résultats montrent que, chez les taurillons subissant un transport de courte durée (inférieure à une heure et demie) entre la ferme et l'abattoir, l'attente à l'abattoir en enclos collectifs provoque 1'apparition de viandes à coupe sombre en proportion élevée. La relation entre fréquence du phénomène et délai séparant le départ de la ferme de l'abattage est extrêmement nette. Il apparaît donc nécessaire, lorsque l'abattoir ne dispose que d'installations de parquage collectif, d'abattre les animaux dès que possible après leur arrivée. Il serait particulièrement intéressant de pouvoir comparer les résultats obtenus par cette méthode et par l'attache individuelle des animaux pendant environ vingt quatre heures avant l'abattage, autre technique également pratiquée. 


\section{Summary}

\section{Effect of preslaughter maintenance of young bulls on the frequency of dark-cutting beef}

The frequency of dark-cutting beef from young bulls subjected to a short lasting transport before slaughter increases with the time elapsed between the departure from the farm and the arrival at the abattoir (fig. I). Mounting induces very high $\mathrm{pH}$ in the meat of mounted animals. Differences between Norman and French Friesian breeds in the percentage of dark-cutting beef were found (Table I). We suggest that young bulls should be slaughtered as soon as possible after their arrival at the slaughterhouse when only collective pens are available.

\section{Références bibliographiques}

Buciter L., I975. Slaughter of meat animals, in ColE P. W., LawriE R. A., Meat, Butterworths, Londres, I33-148.

Fredeen H. T., Martin A. H., Weiss G. M., I974. Changes in tenderness of beef Longissimus dorsi as related to muscle colour and pH. $J$. Fd Sci., 39, 532-536.

LAWRIF, R. A., 1958. Physiological stress in relation to dark-cutting beef. J. Sci. Food Agric., 9, $72 \mathrm{I}-727$.

MoNin G., VAlin C., I976. Influence des conditions d'abattage sur la qualité des viandes de ruminants (particulièrement de bovins). Bull. Techn. C.R.Z.V., Theix, 26, 5-I4.

RHodis D. N., I973. Some observation on the genesis of dark cutting beef from bulls and steers. Meat Research Institute Memorandum, février 1973, Langford.

SoRnay J., I978. Les viandes à pH élevé, un problème préoccupant pour la filière bovine. L'élevage, numéro hors série "La viande bovine ", I4I-I45, Paris.

TARRAN' P. J. V., I975. Post mortem pH and temperature changes in six muscles of beef carcasses. $2 \mathrm{I}$ e Réunion Européenne des Chercheurs en Viande, Berne, 3I aout-5 septembre, I 5-I 7 .

VAlin C., Sornay J., 1975. Contribution à l'étude de la couleur des viandes de taurillon. Publication I.T.E.B., novembre I975, Paris, $3^{8} \mathrm{p}$.

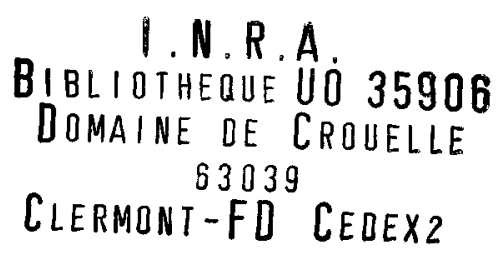

